\title{
Victimización Escolar: Clima Familiar, Autoestima y Satisfacción con la Vida desde una Perspectiva de Género
}

\section{School Victimization: Family Environment, Self-esteem, and Life Satisfaction from a Gender Perspective}

\author{
Amapola Povedano ${ }^{1}$, Leo B. Hendry ${ }^{2}$, Manuel J. Ramos ${ }^{1}$ y Rosa Varela ${ }^{1}$ \\ ${ }^{1}$ Universidad Pablo de Olavide de Sevilla, Spain \\ 2 University of Glamorgan, UK
}

\begin{abstract}
Resumen. En este estudio se analizan en una muestra de adolescentes escolarizados las relaciones entre la percepción del clima familiar y la victimización por los iguales en la escuela junto con la autoestima y la satisfacción con la vida, teniendo en cuenta el género. Las relaciones entre las variables se han analizado en una muestra constituida por 1.884 adolescentes (52\% chicos y $48 \%$ chicas) escolarizados en centros educativos de secundaria con edades comprendidas entre los 11 y los 17 años $(M=13.7, D T=1.4)$. Los resultados del análisis de un modelo de ecuaciones estructurales muestran que la percepción del clima familiar, la autoestima y la satisfacción con la vida se relacionan de forma significativa y negativa con la victimización escolar. Se realizaron análisis multigrupo que no mostraron diferencias significativas entre chicos y chicas, por tanto el modelo general propuesto es equivalente para ambos géneros. Se discuten los resultados obtenidos y sus implicaciones.

Palabras clave: adolescencia, autoestima, clima familiar, género, satisfacción con la vida, victimización.

Abstract. This study analyzed from a gender perspective relationships between perceived family climate, self-esteem, life satisfaction, and adolescent victimization by peers in school. The associations between variables were analyzed with a sample of 1,884 adolescents (52\% boys and $48 \%$ girls), aged 11 through 17 years $(M=13.7, D T=1.4)$. Results structural equation modeling analysis showed that family environment, self-esteem, and life satisfaction were significantly and negatively related to school victimization. Multigroup analyses showed that relationships between variables were not different for boys and girls. We discuss the implications of these results.

Keywords: adolescence, family environement, gender, life satisfaction, self-esteem, victimization.
\end{abstract}

La victimización por los iguales en el contexto escolar, ha sido durante los últimos veinte años foco de atención de un importante grupo de investigadores de las Ciencias Sociales (Espelage y Swearer, 2003; Olweus, 1978; Smith, 2003), y sólo recientemente en España ha emergido como un área relevante de investigación. Este interés en nuestro país, está probablemente asociado a los resultados de estudios pioneros que reflejaban que ser víctima de violencia escolar era un problema que afectaba a numerosos niños y adolescentes escolarizados e invitaban a estudiar en profundidad este problema por sus graves implicaciones (Defensor del Pueblo, 2000). Se considera que un estudiante es víctima de violencia escolar cuando percibe ser objeto permanente de comportamientos violentos realizados por otros estudiantes que no son sus hermanos y que no tienen por qué ser compañeros de clase (Hawker y Boulton, 2000).

Correspondencia: Amapola Povedano Díaz. Departamento de Ciencias Sociales. Universidad Pablo de Olavide. Ctra. Utrera, km. 1. 41013 Sevilla, Spain. E-mail: apovedano@upo.es
La investigación sobre victimización escolar sugiere que estos adolescentes sufren, en muchas ocasiones, graves consecuencias psicológicas y sociales (Defensor del Pueblo, 2007). Así, por ejemplo, estudios previos han documentado cómo la victimización en la escuela está vinculada con la baja autoestima, la ansiedad, el estrés y una valoración negativa de la propia vida (Hodges y Perry, 1999; Hueber, 1991). Las víctimas de violencia escolar tienen un autoconcepto general negativo, una baja satisfacción con la vida (Estévez, Martínez y Musitu, 2006; Prinstein, Boergers y Vernberg, 2001) y un alto grado de infelicidad (Flouri y Buchanan, 2002). En este sentido, algunos autores han sugerido que el que los adolescentes muestren síntomas de infelicidad se podría atribuir al efecto de la victimización, pero también podría ser un factor de riesgo si aquellos que ejercen la violencia perciben que estos estudiantes son blancos fáciles por sus dificultades para defenderse por sí mismos (Cava, Musitu, Buelga y Murgui, 2010; Egan y Perry, 1998).

Existe una larga tradición de investigación psicosocial que muestra que uno de los factores explicativos 
más importantes del comportamiento y desarrollo individual es el contexto social percibido (Bronfenbrenner, 1977; Lewin, 1936). En este sentido, numerosas investigaciones han mostrado que la percepción del contexto familiar y de la calidad de las relaciones entre padres e hijos afectan de manera importante a cómo los niños se convierten en adolescentes y adultos con un adecuado ajuste psicosocial (Maccoby y Martin, 1983; Musitu y García, 2004). Es decir, la percepción de los adolescentes de un clima familiar positivo que fomenta la cohesión, el apoyo, la confianza e intimidad entre los miembros de la familia y promueve dinámicas de comunicación familiar abierta y empática, potencia el ajuste conductual y psicosocial de los adolescentes (Lila y Buelga, 2003, Musitu y Cava, 2003; Musitu, Jiménez y Povedano, 2008).

Los estudios que han tenido en cuenta los efectos de la percepción del clima familiar en la victimización escolar en adolescentes indican una relación directa no significativa entre la victimización escolar en la adolescencia y un clima familiar positivo (Beran y Violato, 2004; Herrero, Estévez y Musitu, 2006; Povedano, Jiménez, Moreno, Amador y Musitu, 2011). Es decir, que a pesar de que el contexto familiar positivo tiene una importante influencia en el desarrollo de los recursos sociales e individuales de los adolescentes (Musitu y Gracia, 2004), no parece ser distinto en adolescentes victimizados y no victimizados. Sin embargo, algunos autores sugieren que un contexto familiar positivo podría estar asociado negativamente con la victimización a través de su influencia en el ajuste psicosocial de los adolescentes (Marturano, Ferreira y D'AvilaBacarji, 2005). En particular, la percepción de dinámicas de relación con los padres fundamentadas en la expresión abierta y positiva de los sentimientos, la cohesión y la ausencia de conflicto se relaciona con altos niveles de autoestima y de satisfacción con la vida de los adolescentes (Hueber, 1991) y estos recursos los podrían proteger de ser víctimizados por sus iguales.

Es importante detenerse a realizar una lectura de estos datos de forma diferencial para chicos y chicas, ya que estudios recientes muestran diferencias de género en estas variables. Así, por ejemplo, para las chicas adolescentes unas relaciones afectivas positivas con sus padres y una adecuada comunicación con ellos, son factores que se han relacionado más estrechamente con la potenciación de su ajuste psicosocial, en comparación con los chicos (Blum, Ireland y Blum, 2003). Estudios previos también indican diferencias significativas por género en relación con la victimización por los iguales, en el sentido de que los chicos están más implicados que las chicas en este tipo de procesos (Lila, Herrero y Gracia, 2008; Seals y Young, 2003). También se ha constatado que la victimización escolar co-ocurre, particularmente entre las chicas, con una baja autoestima (Kling, Hyde, Showers y Buswell, 1999). Sin embargo, la literatura indica que chicos y chicas muestran una satisfacción con la vida similar
(Gilman y Huebner, 2003). No obstante, pocos estudios han examinado conjuntamente la contribución de variables familiares e individuales en la explicación de la victimización por los iguales, analizando también las diferencias de género en estas relaciones.

La literatura científica a la que nos hemos referido sugiere que el clima familiar tiene relaciones positivas con la satisfacción con la vida; que la autoestima se relaciona positivamente con el clima familiar y con la satisfacción vital y que la autoestima tiene una relación directa con la victimización. En este trabajo nos proponemos integrar estas dimensiones de manera conjunta para explicar la victimización escolar desde la perspectiva de género.

En la Figura 1, se expresan las principales hipótesis del estudio. En primer lugar, se esperan encontrar relaciones significativas entre el clima familiar, la autoestima y la satisfacción con la vida. En segundo lugar, se espera encontrar relaciones significativas entre estas dos últimas dimensiones y la victimización escolar. En tercer lugar, y de acuerdo con investigaciones recientes (Beran y Violato, 2004; Herrero et al., 2006) el clima familiar no se relaciona de forma directa con la victimización escolar. Por último, puesto que en trabajos previos se han encontrado diferencias significativas de género en victimización escolar, autoestima y en la percepción de la calidad de las relaciones parento-filiales (Khoury-Kassabri, Benbenishty y Astor, 2004; Kling et al., 1999; Landman-Peeters et al., 2005), en este estudio partimos del supuesto de que las relaciones entre las variables analizadas son significativamente diferentes para los grupos de chicos y chicas adolescentes.

\section{Método}

\section{Muestra}

En este estudio participaron un total de 1884 adolescentes españoles de ambos sexos (52\% varones y $48 \%$ mujeres) de 11 a 17 años $(M=13.7, D T=1.45)$ procedentes de 9 centros educativos españoles (públicos y concertados), ubicados en entornos rurales y urbanos, y distribuidos en $1^{\circ}, 2^{\circ}, 3^{\circ}$ y $4^{\circ}$ de la ESO y $1^{\circ}$ y $2^{\circ}$ de Bachillerato. La muestra es representativa de la comunidad educativa española que tiene un universo poblacional de 2.446.715 estudiantes de ESO y Bachillerato. Se asumió un error muestral de $\pm 2.5 \%$, un nivel de confianza del $95 \%$ y una varianza poblacional de .50 . El tamaño de la muestra requerido fue de 1.536 alumnos. La selección de los participantes se realizó mediante un muestreo estratificado por conglomerados (Santos, Muñoz, Juez y Cortiñas, 2003). Las unidades de muestreo fueron los centros educativos, públicos y concertados de entornos rurales y urbanos de colegios e institutos estatales. Los estratos se establecieron en función de la variable curso. 
Figura 1. Modelo hipotético propuesto en el estudio

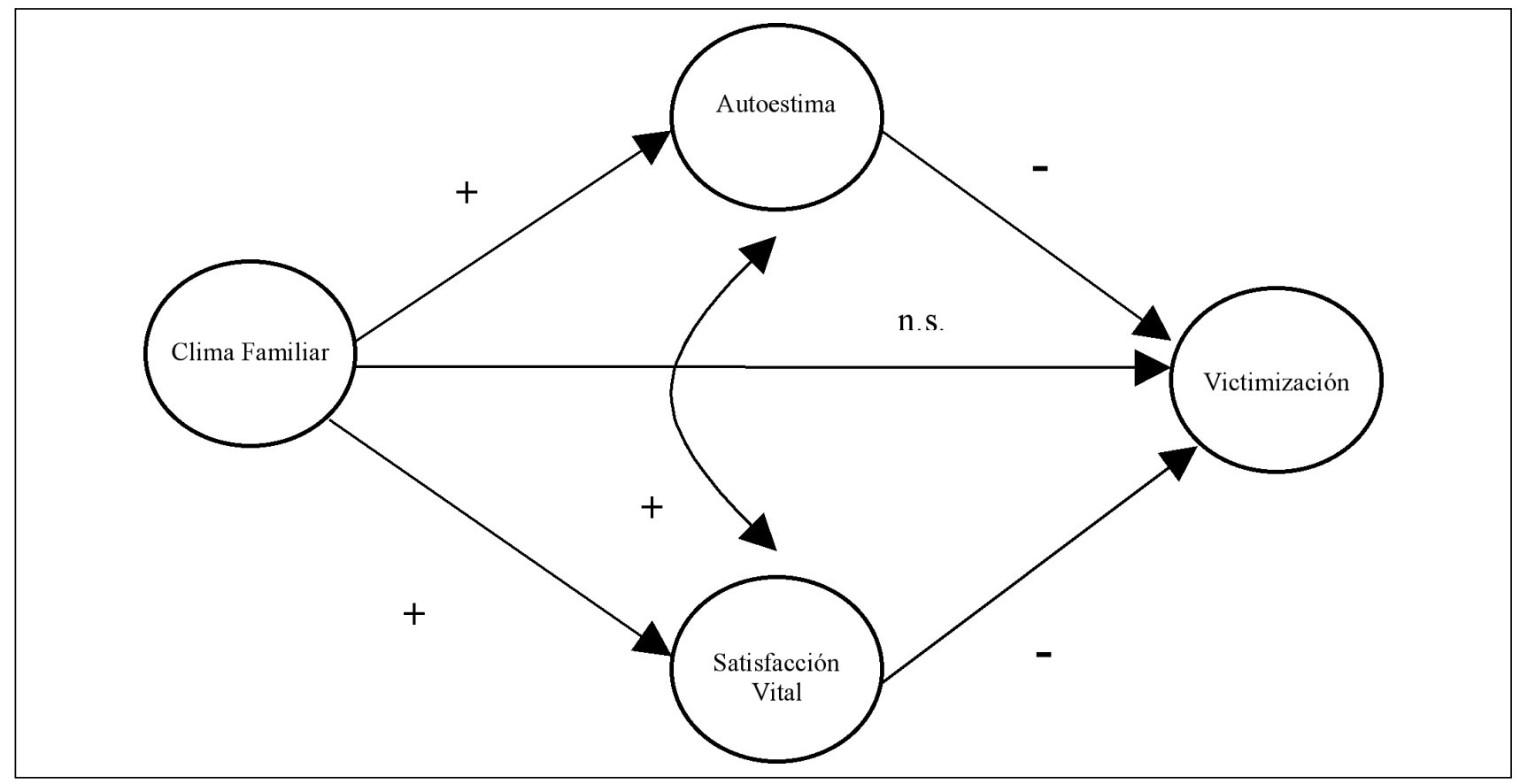

Previamente a la configuración definitiva de la muestra, se llevaron a cabo análisis de diferencias de medias, teniendo en cuenta su ubicación (comunidades autónomas y centros urbanos y rurales) y su gestión (públicos y concertados). Los resultados indican que no existen diferencias significativas entre los centros urbanos y rurales, ni entre centros públicos y concertados, en las variables analizadas en este estudio. Para asegurar la confidencialidad de los participantes, los estudiantes contestaron sólo a determinadas preguntas demográficas que incluían el sexo, la edad y el curso académico.

\section{Procedimiento}

En primer lugar, se envió una carta a los centros educativos seleccionados explicando el proyecto de investigación. Posteriormente se contactó telefónicamente con la dirección de los centros y se concertó una entrevista en la que se explicó el proyecto con detalle, y se entregaron los consentimientos informados para los padres y para el alumnado junto con una carta explicativa de la investigación. Tras la obtención de los permisos correspondientes, se realizó un seminario informativo con la plantilla de profesorado de cada centro donde se explicaron los objetivos y alcance del estudio.

La aplicación de los instrumentos se llevó a cabo por un grupo de investigadores expertos y entrenados. La batería de instrumentos se administró a los adolescentes en sus aulas habituales durante un período regular de clase. El orden de administración de los instru- mentos se contrabalanceó en cada clase y centro educativo. Para aquellos instrumentos en lengua original inglesa se utilizó el método la traducción bidireccional. Se informó en todo momento a los adolescentes que la participación en la investigación era voluntaria y confidencial. El estudio cumplió los valores éticos requeridos en la investigación con seres humanos, respetando los principios fundamentales incluidos en la Declaración Helsinki, en sus actualizaciones, y en las normativas vigentes (consentimiento informado $\mathrm{y}$ derecho a la información, protección de datos personales y garantías de confidencialidad, no discriminación, gratuidad y posibilidad de abandonar el estudio en cualquiera de sus fases).

\section{Instrumentos}

Escala de Clima Familiar (FES; Moos y Moos, 1981; versión española de Fernández-Ballesteros y Sierra, 1989). Esta escala consta de 90 ítems de verdadero-falso, agrupados en 10 subescalas: Cohesión, Expresividad, Conflicto, Independencia, Realización, Orientación Intelectual-Cultural, Orientación Recreativa, Moralidad-Religiosidad, Organización y Control. Estas 10 subescalas se agrupan en tres grandes dimensiones del clima familiar: Relaciones Interpersonales, Crecimiento Personal y Mantenimiento del Sistema. En este estudio, nuestro interés se centró específicamente en las relaciones entre padres e hijos adolescentes, por lo que únicamente se consideraron las tres subescalas que componen la dimensión de Relaciones Interpersonales: Cohesión, que hace referencia al 
grado de compromiso y apoyo familiar percibido por los hijos (ejemplo: "En mi familia, realmente nos ayudamos y apoyamos los unos a los otros"); Expresividad, que hace referencia a la forma en la que los miembros de la familia fomentan la expresión de sus opiniones y sentimientos de forma directa (ejemplo: "En casa hablamos abiertamente de lo que nos parece o queremos"); y Conflicto Familiar, que hace referencia a las relaciones conflictivas entre los miembros de la familia (ejemplo: "En mi familia a veces nos enfadamos tanto que golpeamos o rompemos algo"). En este estudio, el alpha de Cronbach de las subescalas es .86 .73 y .85 respectivamente.

Escala de Autoestima de Rosenberg (Rosenberg, 1989, versión española de Echeburua, 1995). Esta escala ha sido ampliamente utilizada como medida de autoestima y está compuesta por 10 ítems relacionados con sentimientos de auto-aprecio y valía personal (ejemplo: "Creo que tengo numerosas cualidades positivas"). Los ítems se responden en un rango que va desde 1 = muy en desacuerdo hasta $4=$ muy de acuerdo. El alpha de Cronbach en la presente muestra es de 79 .

Escala de Satisfacción con la vida, (Diener, Emmons, Larsen y Griffin, 1985, versión española de Atienza, Pons, Balaguer y García-Merita, 2000). Esta escala está formada por 5 ítems y proporciona un índice general de satisfacción con la vida referido al bienestar subjetivo que el adolescente percibe (ejemplo: "Mi vida es en la mayoría de los aspectos como me gustaría que fuera"). El rango de respuesta oscila de 1 $=$ muy en desacuerdo a $4=$ muy de acuerdo. El alpha de Cronbach obtenido en el presente estudio es de .81 .

Escala de Victimización en la Escuela, basada en Mynard y Joseph (2000). En esta escala los adolescentes indican, en un rango de respuesta de 4 puntos $(1=$ nunca, $4=$ muchas veces), con qué frecuencia durante el último año han experimentado alguna de las experiencias de victimización descritas en los 20 ítems que forman parte del instrumento. Los análisis de investigaciones previas (Cava, Musitu y Murgui, 2007; Jiménez, Musitu, Ramos y Murgui, 2009) de los componentes principales revelaron una estructura de tres factores en el cuestionario: Victimización Relacional, que explica el $49.26 \%$ de la varianza y agrupa 10 ítems (ejemplo, "Algún compañero/a ha contado rumores sobre mi y me ha criticado a mis espaldas"); Victimización Física, que explica el $7.05 \%$ de la varianza y agrupa 4 ítems (ejemplo, “Algún compañero/a me ha pegado o golpeado para hacerme daño de verdad"); y Victimización Verbal, que explica el 5.87\% de la varianza y agrupa 6 ítems (ejemplo, "Algún compañero/a me ha insultado"). Los tres factores obtenidos explican el $62.18 \%$ de la varianza. El alpha de Cronbach para las tres subescalas en esta muestra es $.92, .75$ y .90 respectivamente.

\section{Resultados}

Inicialmente se realizaron análisis correlacionales entre todas las variables objeto de estudio, así como análisis de diferencias de medias en función del género (prueba t). Como se puede observar en la Tabla 1, la expresividad y la cohesión familiar se relacionan negativamente con todas las dimensiones de la victimización, y el conflicto familiar se relaciona positivamente con todas las dimensiones de la victimización. La autoestima general y la satisfacción vital se relacionan positivamente con las dimensiones de expresividad y cohesión familiar y, negativamente, con la dimensión de conflicto. Igualmente, la autoestima general y la

Tabla 1. Correlaciones bivariadas, medias, desviaciones típicas y diferencias entre las medias (en paréntesis) entre las variables objeto de estudio en función del género (chicos a la izquierda de la diagonal y chicas a la derecha en cursiva)

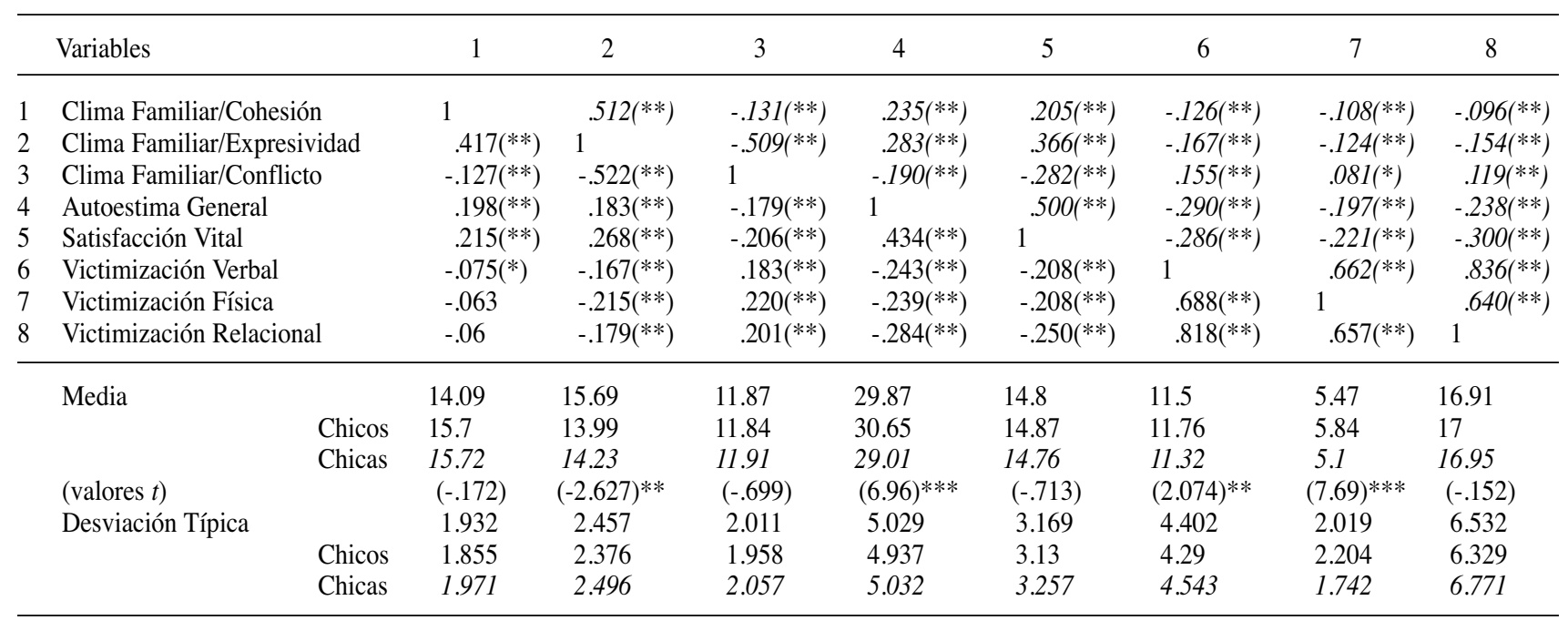

${ }^{*} p<.05 ; * * p<.01 ; * * * p<.001$ 
satisfacción vital se relacionan negativamente con todas las dimensiones de la victimización. También se observan diferencias significativas entre chicos y chicas en expresividad familiar, autoestima y en las dimensiones de la victimización: física y verbal. Las medias de los chicos son superiores a las de las chicas en autoestima y en todas las dimensiones de la victimización que muestran diferencias entre los géneros. Las medias de las chicas son superiores a la de los chicos en expresividad familiar.

Para analizar las relaciones entre las variables se calculó un modelo de ecuaciones estructurales con el programa EQS 6.0 (Bentler, 1995). El modelo calculado tenía los siguientes factores latentes: (1) Clima familiar, compuesto por tres indicadores o variables observables: cohesión, expresividad y conflicto; (2) Autoestima general, compuesto por un único indicador; (3) Satisfacción con la vida, compuesto por un único indicador; (4) Victimización, compuesto por tres variables observables: verbal, física y relacional.

Con el fin de determinar la bondad de ajuste del modelo y la significación estadística de los coeficientes se utilizaron estimadores robustos debido a la desviación de la normalidad de los datos (coeficiente Mardia normalizado: 24.82). El modelo estructural calculado ajusta bien a los datos: $\mathrm{S}-\mathrm{B} \chi^{2}(16, N=1.679)=41.81$, $p<.001 ; \mathrm{CFI}=.99 ; \mathrm{AGFI}=.98 ; \mathrm{GFI}=.99$ y RMSEA $=.03(.02, .05)$. Valores por encima de .95 para los índices CFI, GFI y AGFI y valores por debajo de .05 para RMSEA son indicativos de un buen ajuste (Hu y Bentler, 1999). Este modelo explica el $13 \%$ de la varianza de la variable dependiente, victimización escolar.
La Figura 2 muestra la representación gráfica del modelo estructural final con los coeficientes estandarizados y su probabilidad asociada. Como parte del modelo estructural, se encontró que el clima familiar se relacionaba de forma directa y positiva con la autoestima general $(\beta=.32, p<.001)$ y con la satisfacción con la vida $(\beta=.38, p<.001)$. A su vez, la autoestima y la satisfacción vital estaban asociadas de forma significativa y negativa con la victimización escolar $(\beta=$ $-.14, p<.001 ; \beta=-.23, p<.001$; respectivamente) $\mathrm{y}$ mostraban una fuerte relación positiva entre ambas $(\beta$ $=.40, p<.001)$. Por último, el clima familiar se asociaba de forma directa, negativa y significativa con la victimización escolar $(\beta=-.10, p<.01)$.

Finalmente se efectuó un análisis multigrupo para comprobar la bondad del modelo y la invarianza estructural en función del género (Bentler y $\mathrm{Wu}$, 2002). Para llevar a cabo este propósito se compararon dos modelos: el primer modelo, con constricciones, asume que todas las relaciones entre las variables son iguales para chicos y chicas, mientras que en el segundo modelo, sin constricciones, se estiman todos los coeficientes en ambos grupos. Si al comparar estadísticamente ambos modelos no hay diferencias entre ellos, el modelo con más grados de libertad es el más adecuado. Como se muestra en la Tabla 2, se encontró una diferencia no significativa entre el modelo sin restringir y el restringido $\chi^{2}(11, \mathrm{~N}=$ $1679)=8.82$, n.s. Este resultado apoya la invarianza estructural del modelo en ambos géneros, lo que añade una mayor generalización y aplicabilidad del mismo.

Figura 2. Modelo estructural final con coeficientes de relación y significación estadística

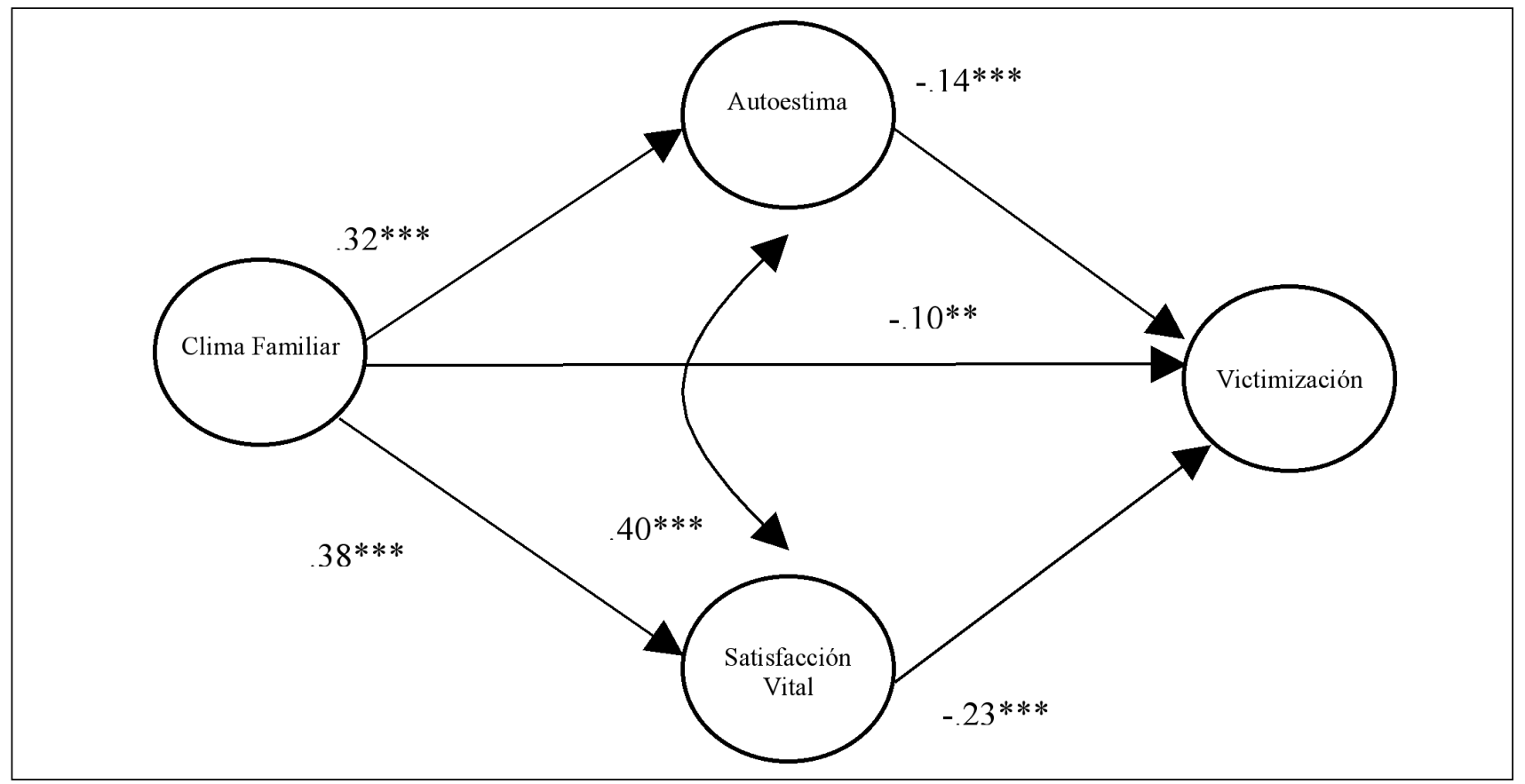

$* p<.05 ; * * p<.01 ; * * * p<.001$ 
Tabla 2. Satorra-Bentler $\chi^{2}$, grados de libertad, probabilidad asociada y comparación de los modelos analizados ${ }^{1}$

\begin{tabular}{|c|c|c|c|c|c|c|c|}
\hline Modelo & Descripción & S-B $\chi^{2}$ & g.l. & $\begin{array}{c}\text { Comparación } \\
\text { Modelos anidados }\end{array}$ & $\begin{array}{l}\text { Diferencia } \\
\text { S-B } \chi^{2}\end{array}$ & $\begin{array}{l}\text { Diferencia } \\
\text { g.l. }\end{array}$ & $p$ \\
\hline Modelo 1 & Modelo con & & & & & & \\
\hline \multirow{3}{*}{ Modelo 2} & Constricciones & 135.74 & 57 & - & - & - & \\
\hline & Modelo sin & & & Modelo 1 & & & \\
\hline & Constricciones & 126.92 & 48 & Modelo 2 & 8.82 & 11 & 0.45 \\
\hline
\end{tabular}

\section{Discusión}

En este estudio se analizaron de forma conjunta las relaciones entre la percepción del adolescente del clima familiar, la autoestima y la satisfacción vital para explicar la victimización escolar desde una perspectiva de género. En primer lugar, nuestros resultados muestran una relación directa, positiva y significativa entre el clima familiar percibido y la autoestima general y la satisfacción con la vida, lo que confirma la primera hipótesis. De esta manera, y en coherencia con otros trabajos (Lila y Buelga, 2003; Musitu y García, 2004) la percepción del adolescente de un clima familiar positivo, que fomenta la cohesión, el apoyo, la confianza e intimidad entre los miembros de la familia y favorece dinámicas de comunicación familiar abierta y empática, potencia el desarrollo de recursos personales en el adolescente, como la autoestima y la satisfacción con la vida.

En este sentido, nuestros resultados sugieren que los adolescentes que muestran recursos sociales y personales ante sus compañeros parecen protegerse de las conductas hostiles de los agresores que buscan como objetivos de violencia a chicos y chicas que se muestran más vulnerables. Así, los resultados del modelo estructural muestran que la autoestima y la satisfacción vital están asociadas de forma significativa y negativa con la victimización escolar, confirmándose nuestra segunda hipótesis, en el sentido de que parece que los adolescentes victimizados perciben su situación en función de sus recursos personales y familiares. Estudios posteriores deberían profundizar en este aspecto y responder a la siguiente pregunta: ¿En qué medida los recursos personales influyen en la percepción de vulnerabilidad y en ser o no víctima del Bullying?

En investigaciones previas se ha mostrado que la victimización escolar no se asocia significativamente con la percepción de relaciones positivas entre padres e hijos (Herrero et al., 2006; Povedano et al., 2011). No obstante, en nuestro estudio los resultados de los análisis no apuntan en esa dirección ya que el clima familiar muestra una relación significativa y negativa, aunque débil, con la victimización escolar. Estos datos desvelan importantes sugerencias acerca del efecto de amortiguación, o buffer, del clima familiar en la victimización escolar. Es decir, la percepción por parte del adolescente de un clima familiar cohesivo, en el que se fomenta el apoyo y la membrecía de los integrantes, con unas relaciones comunicativas abiertas y positivas, parece amortiguar la situación de distrés o malestar psicológico que supone sufrir de forma continuada la violencia escolar en sus diferentes formas, y que como sabemos es una característica determinante de la victimización escolar.

Por último, los datos de este estudio indican diferencias significativas entre los géneros en las variables clima familiar: expresividad y conflicto, autoestima y victimización física y verbal. Estos resultados son consistentes con estudios previos que indican que las chicas tienen una mayor expresividad familiar que los chicos, presentan niveles más bajos de autoestima y tienen, en relación con los chicos, menos probabilidades de sufrir victimización abierta (física y verbal) en la escuela (Pimlott-Kubiak y Cortina, 2003; Seals y Young, 2003). Sin embargo, a pesar de estas diferencias entre los géneros en cuanto a magnitud en las variables señaladas, nuestros resultados muestran que el modelo estructural de relación entre las variables es similar para chicos y chicas, lo que sugiere que el proceso de victimización es equivalente para ambos géneros. Así, puede que las diferencias entre chicos y chicas encontradas en estudios previos sean atribuibles a la mayor visibilidad en algunas de las conductas de los chicos que han sido victimizados, más que a la existencia de diferencias en el proceso de victimización en la escuela (Jiménez et al., 2009).

En síntesis, parece que los adolescentes que pertenecen a familias con un clima familiar positivo desarrollan más recursos personales como una mayor estima (García y Gracia, 2010) y más satisfacción con la vida, y esta circunstancia podría contribuir a que sean menos vulnerables ante la victimización de sus iguales en la escuela. Es decir, los adolescentes que perciben que en sus familias son escuchados y que pueden expresar sus sentimientos y pensamientos sin restricciones, tienen más probabilidad de sentirse más felices con la percepción que tienen de ellos mismos y de sus vidas, mostrando una seguridad en sí mismos que podría protegerles de las agresiones de sus compañeros en la escuela (Estévez, Jiménez y Musitu, 2007). Los resultados de nuestro estudio indican que el clima familiar podría tener un efecto de amortiguación del distrés que sufren los alumnos victimizados lo que sugiere que es importante seguir investigando sobre el posible efecto buffer 
de un clima familiar positivo cuando se estudian los factores explicativos relacionados con la victimización. Además, nuestros resultados indican que estás dinámicas son aplicables tanto a chicas como a chicos adolescentes.

Aunque desde la última década los estudios de género en psicología pueden considerarse de máximo interés (Barberá y Cala, 2009), la investigación sobre violencia escolar que incluye la perspectiva de género es aún escasa. En esta investigación se ha tenido en cuenta el género de los adolescentes, sin embargo cuenta con la limitación de no tomar en consideración la construcción del género como una variable. En este sentido, el presente trabajo se ha desarrollado bajo la aproximación de las diferencias entre-sexos, pero también bajo el análisis del género como una herramienta analítica (Stewart y McDermott, 2004), profundizando en el conocimiento de variables marcadas por el género como son la autoestima y la victimización de los adolescentes. Otra limitación del presente estudio es que está basado en un diseño transversal, por lo que no es posible establecer relaciones causales. Por último, creemos que es necesario continuar explorando estas relaciones en función del género ya que en el presente trabajo no se ha diferenciado la victimización escolar en cuanto a formas y contenido lo que consideramos como una debilidad del estudio.

A pesar de estas limitaciones, creemos que los resultados obtenidos contribuyen a un mejor conocimiento de las relaciones entre el clima familiar y la victimización por los iguales en la escuela. Un contexto familiar fundamentado en la expresividad abierta de opiniones, sentimientos y afectos, en el apoyo y la cohesión y con ausencia de fuertes conflictos proporciona al adolescente los recursos individuales y sociales necesarios para mejorar sus relaciones de convivencia y su ajuste en el contexto escolar, además de protegerlo de potenciales amenazas. Finalmente, destacaríamos de una manera especial la trascendencia de seguir explorando cómo el clima familiar podría tener un efecto buffer, o de amortiguación, sobre el malestar psicológico que sufren muchos adolescentes ante la experiencia de la victimización escolar, un aspecto que está muy poco explorado.

\section{Agradecimientos}

Esta investigación se ha elaborado en el marco del Proyecto de Investigación PSI2008-01535/PSIC "Violencia escolar, victimización y reputación social en la adolescencia", subvencionado por el Ministerio de Educación y Ciencia de España y del Proyecto de Investigación Educativa 2009 PIV-015/09 "Violencia y victimización en la adolescencia: análisis desde una perspectiva de género", subvencionado por la Consejería de Educación de la Junta de Andalucía, también está cofinanciado por los Fondos Europeos FEDER.

\section{Referencias}

Atienza, F. L., Pons, D., Balaguer, I. y García-Merita, M. (2000). Propiedades psicométricas de la Escala de Satisfacción con la Vida en adolescentes. Psicothema, 12, 314-319.

Barberá, E. y Cala, M. J. (2008). Perspectiva de género en la psicología académica española. Psicothema, 20, 236-242.

Bentler, P. M. (1995). EQS structural equations program manual. Encino, CA: Multivariate Software.

Bentler, P. y Wu, E. (2002). EQS 6 for windows user's guide. Encino, CA: Multivariate Software.

Beran, T. y Violato, C. (2004). A model of childhood perceived peer harassment: Analyses of the Canadian national longitudinal survey of children and youth data. The Journal of Psychology, 138, 129-147.

Bronfenbrenner, U. (1977). Toward an ecology of human development. American Psychologist, 32, 513-531.

Blum, J., Ireland, M. y Blum, R. W. (2003). Gender differences in juvenile violence: A report from Add Health. Journal of Adolescent Health, 32, 234-240.

Cava, M. J., Musitu, G., Buelga, S. y Murgui, S. (2010). The relationships of family and classroom environments with peer relational victimization: An analysis of their gender differences. The Spanish Journal of Psychology, 13, 156165.

Cava, M. J., Musitu, G. y Murgui, S. (2007). Individual and social risk factors related to overt victimization in a sample of Spanish adolescents Psychological Reports, 101, 275-290.

Defensor del Pueblo (2000). Informes estudios y documentos. Violencia escolar: el maltrato entre iguales en la educación secundaria obligatoria. Madrid: Defensor del Pueblo.

Defensor del Pueblo (2007). Violencia escolar: el maltrato entre iguales en la educación secundaria obligatoria, 1999-2006. Madrid: Defensor del Pueblo.

Diener, E. D., Emmons, R. A., Larsen, R. J. y Griffin, S. (1985). The Satisfaction With Life Scale. Journal of Personality Assessment, 49, 71-75.

Egan, S. K. y Perry, D. G. (1998). Does low self-regard invite victimization? Developmental Psychology, 34, 299-309.

Estévez, E., Jiménez, T. I. y Musitu, G. (2007). Relaciones entre padres e hijos adolescentes. Nau Llibres: Valencia.

Estévez, E., Martínez, B. y Musitu, G. (2006). La autoestima en adolescentes agresores y víctimas en la escuela: La perspectiva multidimensional. Intervención Psicosocial, 15, 223-233.

Espelage, D. L. y Swearer, S. M. (2003). Research on school bullying and victimization: What have we learned and where to we go from here? School Psychology Review, 32, 365-383.

Fernández-Ballesteros, R. y Sierra, B. (1989). Escalas de Clima Social FES, WES, CIES y CES. Madrid: TEA.

Flouri, E. y Buchanan A. (2002). Life satisfaction in teenage boys: The moderating role of father involvement and bullying. Aggressive Behavior, 28, 126-133.

García, F. y Gracia, E. (2010). ¿Qué estilo de socialización parental es el idóneo en España? Un estudio con niños y 
adolescentes de 10 a 14 años. Infancia y Aprendizaje, 33, 365-384.

Gilman, R. y Huebner, S. (2003). A review of life satisfaction research with children and adolescents. School Psychology Quarterly, 18, 192-205.

Hawker, D. S. J. y Boulton, M. J. (2000). Twenty years' research on peer victimization and psychosocial maladjustment: a meta-analytic review of cross-sectional studies. Journal of Child Psychology and Psychiatry, 41, 441455.

Herrero, J., Estévez, E. y Musitu, G. (2006). The relationships of adolescent school-related deviant behaviour and victimization with psychological distress: Testing a general model of the mediational role of parents and teachers across groups of gender and age. Journal of Adolescence, 29, 671-690.

Hodges, E. V. E. y Perry, D. G. (1999). Personal and interpersonal antecedents and consequences of victimization by peers. Journal of Personality and Social Psychology, 76, 677-685.

Hu, L. y Bentler, P. M. (1999). Cutoff criteria for fit indexes in covariance structure analysis: conventional criteria versus new alternatives. Structural Equation Modeling, 6, $1-55$.

Huebner, E. S. (1991). Correlates of life satisfaction in children. School Psychology Quarterly, 6, 103-111.

Jiménez, T. I., Musitu, G., Ramos, M. J. y Murgui, S. (2009). Comunity involvement and victimization at school: an análisis through family, personal and social adjustment. Journal of Community Psychology, 37, 959-974.

Khoury-Kassabri, M., Benbenishty, R. y Astor, R. (2004). The contributions of community, family, and school variables to student victimization. American Journal of Community Psychology, 34, 187-204.

Kling, K. C., Hyde, J. S., Showers, C. J. y Buswell, B. N. (1999). Gender differences in self-esteem: A meta-analysis. Psychological Bulletin, 125, 470-500.

Landman-Peeters, K., Hartman, C., Van der Pompe, G., Den Boer, J., Minderaa, R. y Ormel, J. (2005). Gender differences in the relation between social support, problems in parent-offspring communication, and depression and anxiety. Social Science \& Medicine, 60 , 2549-2560.

Lewin, K. (1936). Principles of topological psychology. New York: McGraw-Hill.

Lila, M. S. y Buelga, S. (2003). Familia y adolescencia: el diseño de un programa para la prevención de conductas de riesgo. Encuentros en Psicología Social, 1, 72-78.

Lila, M. S., Herrero, J. y Gracia, E. (2008). Multiple victimization of Spanish adolescents: A multilevel analysis. Adolescence, 43, 333-350.

Maccoby, E. E. y Martin, J. A. (1983). Socialization in the context of the family: parent-child interaction. En P.H. Mussen (Ed.), Handbook of child psychology (pp. 1-101). New York: Wiley.
Marturano, E. M., Ferreira, M. C. T. y D’Avila-Bacarji, K. M. G. (2005). An evaluation scale of family environment for the identification of children at risk of school failure. Psychological Reports, 96, 307-321.

Moos, R. H. y Moos, B. S. (1981). Family Environment Scale manual. Palo Alto, CA: Consulting Psychologist Press.

Musitu, G. y Cava, M. J. (2003). El rol del apoyo social en el ajuste de los adolescentes. Intervención Psicosocial, 12, 179-192.

Musitu G. y García, F. (2004). Consecuencias de la socialización familiar en la cultura española. Psicothema, 16, 288-293.

Musitu, G., Jiménez, T. I. y Povedano, A. (2008). Familia y escuela: escenarios de riesgo y protección en la violencia escolar. En A. Carpi, C. Gómez, C. Guerrero y F. Palmero (Coords), Violencia y Sociedad (pp. 59-99). Generalitat Valenciana. Conselleria D'Educació.

Mynard, H. y Joseph, S. (2000). Development of the Multidimensional Peer-Victimization Scale. Aggressive Behavior, 26, 169-178.

Olweus, D. (1978). Aggression in the schools: bullies and whipping boys. Washington DC: Hemisphere.

Pimlott-Kubiak, S. y Cortina, L. M. (2003). Gender, victimization, and outcomes: Reconceptualizing risk. Journal of Consulting and Clinical Psychology, 71, 528-539.

Povedano, A, Jiménez, T. I., Moreno, D., Amador, L. y Musitu, G. (2011). Relación del conflicto y la expresividad familiar con la victimización en la escuela: El rol de la autoestima, la sintomatología depresiva y el género de los adolescentes. (En revisión)

Prinstein, M. J., Boergers, J. y Vernberg, E. M. (2001). Overt and relational aggression in adolescents: Socialpsychological adjustment of aggressors and victims. Journal of Clinical Child Psychology, 30, 479-491.

Rosenberg, M. (1989). Society and the adolescent selfimage. (Rev. ed.) Middletown, CT: Wesleyan Univer. Press.

Santos, J., Muñoz, A., Juez, P. y Cortiñas, P. (2003). Diseño de encuestas de estudio de mercado. Técnicas de muestreo y análisis multivariante. Madrid: Fundación Ramón Areces.

Satorra, A. y Bentler, P. M. (1994). Corrections to test statistics and standard errors in covariance structure analysis. En A. von Eye y C.C. Clogg (Eds.), Latent variable analysis: Applications for developmental research (pp. 399-419). Thousand Oaks, CA: Sage.

Seals, D. y Young, J. (2003). Bullying and victimization: prevalence and realtionship to gender, grade level, ethnicity, self-esteem, and depression. Adolescence, 38, 735747.

Smith, P. K. (Ed.). (2003). Violence in schools: The response in Europe. London: Routledge Falmer.

Stewart, A. J. y McDermott, C. (2004). Gender in psychology. Annual Review of Psychology, 55, 519-544.

Manuscrito Recibido: 08/06/2010

Revisión Recibida: 13/10/2010

Manuscrito Aceptado: 18/10/2010 